\title{
Estimativa da Sensibilidade ao Contraste EspaCial de LuminÂNCIA E DisCRIMINAÇão DE Cores por meio do Potencial Provocado Visual TRANSIENTE ${ }^{1}$
}

\section{Bruno Duarte Gomes ${ }^{2}$, Givago da Silva Souza², Anderson Raiol Rodrigues $^{2}$, Cézar Akiyoshi Saito ${ }^{2}$, Manoel da Silva Filho ${ }^{3}$ e Luiz Carlos de Lima Silveira ${ }^{4}$}

Universidade Federal da Pará - UFPA

O potencial provocado visual (VEP) é uma resposta cortical registrável na superfície do couro cabeludo, que reflete a atividade dos neurônios de V1. É classificado, a partir da freqüência temporal de estimulação, em transiente ou de estado estacionário. Outras propriedades do estímulo parecem provocar uma atividade seletiva dos diversos grupos de neurônios existentes em V1. Desse modo, o VEP vem sendo usado para estudar a visão humana acromática e cromática. Diversos trabalhos usaram o VEP para estimar a sensibilidade ao contraste de luminância no domínio das freqüências espaciais. Mais recentemente, há estudos que empregaram o VEP para medir os limiares de discriminação de cores. O VEP transiente pode complementar as medidas psicofísicas de sensibilidade ao contraste espacial de luminância e de discriminação cromática, e constitui um método não invasivo para estudar a visão de indivíduos com dificuldades de realizar testes psicofísicos.

Descritores: Discriminação de cores. Sensibilidade de contraste (visão).

Potenciais visuais provocados. Estimulação visual.

1 Apoio financeiro do CNPq-PRONEX/SECTAM-FUNTEC/FADESP, CAPES, CAPESPROCAD e FINEP (IBN-Net - Rede Instituto Brasileiro de Neurociências).

2 Pós-Graduandos do Departamento de Fisiologia do Centro de Ciências Biológicas UFPA. Endereços eletrônico do primeiro autor: brunodgomes@yahoo.com.br

3 Docente do Departamento de Fisiologia do Centro de Ciências Biológicas - UFPA.

4 Docente do Departamento de Fisiologia do Centro de Ciências Biológicas - UFPA. Diretor Geral do Núcleo de Medicina Tropical - UFPA. 
B. D. Gomes, G. S. Souza, A. R. Rodrigues, C. A. Saito, M. Silva-Filho e L. C. L. Silveira

\section{O potencial provocado visual}

O potencial provocado visual ou VEP (Visual Evoked Potential) consiste da combinação de potenciais pós-sinápticos de neurônios localizados principalmente no córtex visual primário (V1), gerados durante a ocorrência de estímulos visuais (Whittaker \& Siegfried, 1983). O VEP reflete em grande medida o registro da atividade da região retiniana central, pois o mapa retinotópico da projeção dessa região em V1 sofre enorme magnificação devido à sua grande densidade neuronal (e.g. Silveira, Picanço-Diniz, Sampaio, \& Oswaldo-Cruz, 1989). Nessa região retiniana, as chamadas "linhas privadas" compreendem conexões entre fotorreceptores, células bipolares e células ganglionares na proporção de 1:1:1. Com isso, mais de 50\% das células de V1 têm campos receptivos localizados nos $10^{\circ}$ centrais do campo visual. Além de ser um método de escolha para estudar a região central da visão, aquela de grande importância oftalmológica, o VEP tem ainda a vantagem de ser um método não invasivo para o estudo das vias retino-tálamo-corticais (Regan \& Spekreijse, 1986).

O VEP tem amplitude da ordem de milivolt quando registrado diretamente no córtex cerebral, mas é reduzido à escala de microvolt quando captado por eléctrodos de superfície posicionados sobre o couro cabeludo. Essa queda de tensão ocorre entre os diversos meios (meninges, osso, músculo, pele) que se interpõem entre a origem do potencial e o local de registro, atenuando a amplitude do sinal.

O registro eletrofisiológico do VEP em seres humanos é uma variação da eletroencefalografia (EEG), chamada de encefalografia relacionada a eventos, e é realizado usando-se eletródios de superfície com resistência de contato entre 3 e $5 \mathrm{KOhm}$, colocados em pontos estratégicos na superfície do crânio (Odom, et al., 2004). A diferença de potencial entre os eletródios é captada por amplificadores diferenciais de elevada impedância - termo mais correto, porque a via de passagem da corrente sofre influência de outros opositores, não só de componentes puramente resistivos (até 1014 Ohm) (Hjorth, 1975).

Em virtude da sua reduzida amplitude, o registro do VEP está associado a ruídos elétricos das mais variadas fontes, principalmente aquele proveniente da rede elétrica. Atualmente, os sistemas que pretendem registrar potenciais elétricos dessa ordem de magnitude, já trazem incorporados aos seus circuitos 
Estimativa da Sensibilidade ao Contraste Espacial de Luminância e Discriminação...

eletrônicos, filtros digitais ou até condicionadores de sinais dedicados à execução dessa tarefa, sem a necessidade de circuitos externos adicionais, melhorando substancialmente a relação sinal / ruído. Tais sistemas são conectados a microcomputadores e gerenciados por programas específicos para a realização do registro, sob essas condições, permitindo que o avaliador ajuste seu protocolo de estimulação / registro para as diversas fases que o teste impõem.

\section{Tipos de VEP}

O VEP pode ser classificado em dois tipos, VEP transiente e VEP de estado estacionário, classificação essa decorrente das condições de estimulação visual. O VEP transiente é gerado por estímulos intervalados por grandes períodos de tempo, permitindo ao cérebro retornar a um estado de repouso entre as apresentações. Ou seja, o VEP transiente é produzido pela apresentação de estímulos com frequiência temporal baixa, o que permite a caracterização da resposta provocada no domínio do tempo, sendo possível medir a amplitude e a latência (ou tempo implícito) de cada resposta provocada (Regan, 1982; Regan \& Spekreijse, 1986).

O VEP de estado estacionário, por outro lado, é produzido por uma frequiência temporal de estimulação elevada, impedindo que os neurônios se recuperem da atividade provocada pela estimulação imediatamente anterior, resultando em uma resposta semelhante a uma senóide que, na verdade, é o produto da superposição das diversas respostas vinculadas à estimulação (Regan, 1966, 1982; Regan \& Spekreijse, 1986; Tobimatsu, Kurita-Tashima, Nakayama-Hiromatsu, \& Kato, 1993). No VEP estacionário as grandezas de interesse são amplitude e fase da resposta.

O emprego de um ou outro tipo de VEP é função do que se quer avaliar nas respostas. No primeiro caso, obtêm-se respostas relativas a uma função impulso, cuja principal característica é o grau de espalhamento da energia ao longo de momentos sucessivos da estimulação. No segundo caso, as respostas estão relacionadas a uma função de transferência de frequiências, passível de decomposição numa função de transferência de amplitude e de transferência de fase. 
A análise da amplitude e da latência é considerada uma grande vantagem do VEP transiente porque permite correlacionar diretamente as características temporais do estímulo com o surgimento ou alteração da resposta. A desvantagem desse método é que pode ser difícil identificar componentes homólogos entre diferentes indivíduos ou condições de estimulação. O VEP de estado estacionário é mais facilmente descrito em termos quantitativos por que é composto por um número específico de componentes de frequiência que podem ser caracterizados em amplitude e fase pelo uso da transformada rápida de Fourier (Fast Fourier Transform, FFT) (Bracewell, 1986; Regan, 1966, 1982).

\section{Importância da estimulação}

A percepção de uma cena visual é obtida pelo funcionamento integrado de neurônios das vias retino-tálamo-corticais capazes de representar na sua atividade as variações de contraste espaçotemporal entre os diferentes pontos dessa cena e de refletância espectral da superfície dos objetos que a compõem. As características dos estímulos visuais capazes de atuar nessas células constituem o ponto de partida para o entendimento das respostas visuais provocadas.

Os protocolos de estimulação são montados a partir do que se conhece da teoria de análise de sistemas e dos resultados de um grande conjunto de estudos eletrofisiológicos e psicofísicos. Por exemplo, para o estudo da visão no domínio das freqüências utiliza-se estímulos compostos por variações senoidais no espaço (faixas que variam em luminância ou cromaticidade senoidalmente ao longo de certa extensão espacial, como a da tela de um monitor CRT) ou no tempo (variações senoidais de luminância ou cromaticidade em momentos sucessivos do tempo). Esse tipo de estímulo é matematicamente a forma mais simples de testar um sistema nesses domínios.

As redes senoidais no domínio espectral espacial podem ser caracterizadas pela frequiência espacial, pela orientação, pelo contraste e pela fase espacial. A facilidade de manipular cada um dos elementos dessas redes permite estudar como o sistema nervoso responde às mudanças dos valores desses elementos e caracterizar como a nossa visão processa informação nesse domínio. Assim, através da eletrofisiologia e psicofísica tem sido observado que os neurônios do sistema visual e o sistema visual como um todo respondem 
Estimativa da Sensibilidade ao Contraste Espacial de Luminância e Discriminação...

seletivamente às características específicas das redes senoidais. Por exemplo, no córtex visual primário existem neurônios que só respondem a uma determinada faixa de freqüências espaciais, orientações, contrastes e fases espaciais, de tal forma que o sistema visual opera aproximadamente como um analisador de freqüências espaciais (Campbell \& Robson, 1968; De Valois, Yund, \& Hepler, 1982).

O pesquisador determina que grupos de neurônios quer estimular de acordo com seus objetivos, ou seja, com as funções visuais que pretende explorar e quantificar. Quantos menos elementos da imagem são variados ao longo dos experimentos, mais seletiva será a estimulação quanto ao grupo de células do sistema visual e, nesse caso, os estímulos são de configurações simples, como redes senoidais isocromáticas ou isoluminantes (e.g.: Gomes, Souza, Rodrigues, Saito, Silveira, \& da Silva Filho, 2006). No entanto, caso a pretensão seja estimular o maior número de células possíveis, o estímulo deve apresentar um padrão complexo de variação de luminância ou cor, como redes quadradas ou de outros perfis não senoidais (e.g.: Odom et al., 2004).

\section{Estimativa da sensibilidade ao contraste espacial de luminância através do VEP}

A avaliação da visão espacial acromática baseia-se na capacidade de diferenciar a quantidade de luz entre duas áreas adjacentes, ou seja, alguma forma de medir o número de fótons oriundos de locais próximos no campo visual. No domínio das frequiências, corresponde à avaliação da sensibilidade ao contraste de luminância numa determinada freqüência espacial. O menor contraste perceptível é chamado de contraste limiar. A sensibilidade ao contraste é definida como a recíproca do contraste limiar. A função que expressa a sensibilidade em diferentes freqüências espaciais é denominada de função de sensibilidade ao contraste (FSC) espacial de luminância.

Os diversos aspectos do estímulo estão representados eletrofisiologicamente nas características do VEP. Por exemplo, existe uma boa correlação entre o contraste do estímulo e a amplitude do VEP, de tal forma que quanto maior o contraste, maior a amplitude de diversos componentes da resposta cor- 
tical (Campbell \& Maffei, 1970; Souza, 2005; Souza, Gomes, Saito, Silveira, $\&$ da Silva Filho, 2006). Além disso, há uma boa correlação entre as medidas psicofísicas e eletrofisiológicas usando o VEP para vários parâmetros, como os limiares de sensibilidade ao contraste (Campbell \& Maffei, 1970; Souza, 2005; Souza, et al., 2006). Entretanto, essa correlação segue funções algumas vezes complexas devido, principalmente, à intrusão de diversos tipos de não linearidades, como acontece em níveis supralimiares de contraste (Campbell $\&$ Kulikowiski, 1972; Souza, 2005; Souza et al., 2006; Strasburger, Rentschler, \& Scheidler, 1986).

Campbell e Maffei (1970) foram os primeiros a utilizar o VEP como uma ferramenta importante para estimar a sensibilidade ao contraste espacial de luminância. Eles estimularam o sistema visual com redes senoidais que sofriam reversão de fase de $180^{\circ}$ à freqüência temporal de $8 \mathrm{~Hz}$. Eles observaram uma diminuição na amplitude do VEP em função do logaritmo do contraste. O contraste limiar foi estimado extrapolando-se a função até o contraste no qual a amplitude era zero. A sensibilidade ao contraste espacial de luminância encontrada eletrofisiologicamente foi igual àquela obtida psicofisicamente em uma faixa de frequiências espaciais entre 1,5 - $35 \mathrm{cpg}$. Um importante achado do trabalho de Campbell e Maffei (1970) foi que a linearidade da função amplitude do VEP versus log do contraste não era observada para todas as frequiências espaciais e nem para todos os níveis de contrastes. Eles encontraram uma função de dupla tendência de decaimento da amplitude versus log contraste em freqüências espaciais abaixo de $3 \mathrm{cpg}$, e procuraram explicar esse fenômeno pela contribuição simultânea de diferentes regiões retinianas, foveal e parafoveal, para a resposta cortical.

Harris, Atkinson e Braddick (1976) estudando a sensibilidade ao contraste espacial de luminância encontraram boa correlação, nas frequiências espaciais baixas, entre os valores obtidos pelo VEP de estado estacionário (reversão de fase de $180^{\circ}$, freqüencia temporal de $10 \mathrm{~Hz}$ ) e por métodos psicofísicos. No entanto, em frequiências espaciais intermediárias e altas, os valores de sensibilidade ao contraste derivados da eletrofisiologia eram menores que os obtidos pela psicofísica.

Cannon (1983a) estimou a FSC através do emprego da estimulação visual com redes senoidais espaciais com dois tipos de apresentação, reversão 
Estimativa da Sensibilidade ao Contraste Espacial de Luminância e Discriminação...

de fase de $180^{\circ}$ e onset / offset. Ele comparou as FSCs eletrofisiológicas com aquelas obtidas psicofisicamente com o mesmo tipo de estimulação e observou que, na modulação temporal reversa, o formato da FSC obtida pelo VEP era semelhante à FSC psicofísica, enquanto que na estimulação onset / offset, a FSC eletrofisiológica apresentou valores bem menores para a sensibilidade ao contraste em frequiências espaciais baixas do que a FSC psicofísica.

Cannon (1983b) empregando VEP de estado estacionário avaliou a influência do tamanho da área do campo visual estimulado sobre a FSC. Ele mostrou que o aumento dessa área eleva os valores de sensibilidade ao contraste espacial de luminância nas frequiências espaciais intermediárias e baixas, tanto empregando o VEP quanto a psicofísica como procedimento de avaliação. A diferença residia nos valores mais altos de sensibilidade ao contraste na psicofísica, assim como numa maior atenuação da sensibilidade ao contraste nas frequiências espaciais baixas com a diminuição da área na eletrofisiologia. Ele também estudou a FSC na região parafoveal, observando novamente valores de sensibilidade ao contraste psicofísicos maiores que os eletrofisiológicos.

Diversos outros trabalhos também avaliaram a FSC utilizando o VEP de estado estacionário com o procedimento de varredura (Chen, $\mathrm{Wu}, \& \mathrm{Wu}$, 1990; Lopes de Faria, Katsumi, Arai, \& Hirose, 1998; Norcia, Tyler, Hamer, \& Wesemann, 1989; Orban \& Orban, 1985; Seiple, Kupersmith, Nelson, \& Carr, 1984; Strasburger, Remky, Murray, Hadjizenonos, \& Rentschler, 1996). Nesse procedimento, chamado VEP estacionário de varredura, apresenta-se inicialmente um estímulo de determinada freqüência espacial e alto contraste. Em seguida, abaixa-se rapidamente o contraste até a resposta eletrofisiológica desaparecer dentro do ruído não correlacionado com a estimulação. Este método permite que o tempo total de registro seja menor que nas configurações tradicionais, transiente e estacionário. Com exceção do trabalho de Chen et al. (1990), que encontrou valores de sensibilidade ao contraste semelhantes entre os métodos eletrofisiológico (com esse procedimento) e psicofísico, os demais trabalhos obtiveram valores menores com o VEP de varredura comparados às estimativas psicofísicas. Oliveira, Costa, de Souza e Ventura (2004) através do VEP estacionário de varredura estimaram a sensibilidade ao contraste de recém-nascidos saudáveis, comparando os prematuros com aqueles nascidos a termo. Eles observaram que a sensibilidade ao contraste em $4 \mathrm{cpg}$ aumentava 
B. D. Gomes, G. S. Souza, A. R. Rodrigues, C. A. Saito, M. Silva-Filho e L. C. L. Silveira

no período entre 3 e 10 meses, enquanto a sensibilidade ao contraste em 0,2 cpg mantinha-se inalterada no mesmo período.

O VEP de estado estacionário é muito mais utilizado do que o VEP transiente, uma vez que sendo a estimulação realizada em frequiência temporal mais alta, o tempo total de registro é bem menor. Esse tempo ainda é consideravelmente otimizado quando o VEP estacionário é realizado com o procedimento de varredura. Em virtude disso, poucos trabalhos sobre a sensibilidade ao contraste têm sido feitos com o VEP transiente. Katsumi, Tanino e Hirose (1985), utilizando estimulação por reversão de fase de $180^{\circ}$ à frequiência temporal de $2 \mathrm{~Hz}$, obtiveram valores menores para a sensibilidade ao contraste com o VEP transiente quando comparados com aqueles obtidos psicofisicamente.

Uma possível explicação para as diferenças encontradas por vários autores entre os valores de sensibilidade ao contraste obtidos com métodos eletrofisiológicos e psicofísicos, reside na chamada "Doutrina do Neurônio" do neurofisiologista inglês Horace Basil Barlow (1921-). Barlow desenvolveu essa hipótese a partir da estrutura estatística das mensagens sensoriais e das evidências experimentais mostrando que os neurônios isolados nas vias sensoriais são altamente seletivos e sensíveis e que, portanto, as discriminações perceptuais podem ser baseadas diretamente na atividade de um pequeno número dos neurônios mais ativos numa dada condição de estimulação (Barlow, 1972). Portanto, os eventos sensoriais seriam representados no cérebro através de um código esparso, onde a atividade de um número pequeno de neurônios selecionados, chamados "células cardinais" forma uma representação distribuída do que ocorre na entrada sensorial (Barlow, 1972). Assim, é possível que enquanto poucos neurônios sejam necessários para que a percepção consciente ocorra e o sujeito responda positivamente num experimento psicofísico, por outro lado é também provável que sejam necessários muitos neurônios disparando cooperativamente para produzir uma resposta mensurável por eletroencefalografia de eventos e discriminável contra o ruído bioelétrico concomitantemente registrado (Katsumi et al., 1985).

Souza e colaboradores avaliaram a sensibilidade ao contraste com VEP transiente (reversão de fase de $180^{\circ}$ à $1 \mathrm{~Hz}$ de freqüência temporal) (Figura 1A) e compararam com a sensibilidade ao contraste avaliada por métodos psicofísicos em duas condições de estimulação, dinâmica (mesma condição de 
estimulação que a usada na eletrofisiologia) e estática (Souza, 2005; Souza et al., 2006). Eles observaram que a FSC eletrofisiológica apresenta maior similaridade com FSC psicofísica obtida em condições semelhantes de estimulação, especialmente nas frequiências espaciais baixas e intermediárias (Figura 1D). Já nas freqüências espaciais altas, a sensibilidade ao contraste foi sempre maior nos procedimentos psicofísicos. Neste trabalho, assim como outros com humanos e primatas não humanos (Bobak, Bodis-Wollner, Harnois, \& Thornton, 1984; Bonds, Casagrande, Norton, \& Debruyn, 1987; Campbell \& Maffei, 1970; Chen et al., 1990; Katsumi et al., 1985; Nakayama \& Mackeben, 1982; Rudvin, Valberg, \& Kilavik, 2000; Strasburger et al., 1996), foram observadas não linearidades na relação entre a amplitude do VEP e o logaritmo do contraste, tanto a saturação em contrastes altos como o decaimento em dupla tendência ao longo da faixa de contrastes visíveis (Figura 1B-C, respectivamente). Analisando esses achados, Souza e colaboradores procuraram explicá-los pela contribuição diferencial das diversas vias visuais para o VEP: em frequiências espaciais baixas o VEP seria dominado pela contribuição da via magnocelular (via $\mathrm{M}$ ) em todos os níveis de contrastes; em freqüências espaciais intermediárias e altas, a via $\mathrm{M}$ continuaria a contribuir majoritariamente para o VEP eliciado por estímulos de contrastes baixos, enquanto que para contrastes altos, várias vias seriam estimuladas, inclusive a via parvocelular (via P). Essa interpretação é semelhante àquela utilizada por Bobak et al. (1984) e Rudvin et al. (2000) para explicar seus próprios resultados e está de acordo com o que é conhecido sobre as propriedades das células M e P (Kaplan \& Shapley, 1986).

\section{VEP obtido com estímulos cromáticos}

O VEP é um procedimento importante para o estudo da resposta do sistema visual humano ao contraste cromático puro. Essa resposta é produzida pela atividade das vias de oponência de cores verde-vermelha (via P) e azulamarela (via koniocelular ou via K), as quais são anatômica e fisiologicamente distintas da via magnocelular ou via $\mathrm{M}$, que veicula informação visual correspondente ao contraste acromático (Hendry \& Reid, 2000; Sincich \& Horton, 2005). As três vias formam-se na retina, onde ocorrem os primeiros estágios do processamento visual e compreendem classes específicas de células gangliona- 
Figura 1. A. VEP transiente obtido com estimulação com redes senoidais, freqüência espacial de 2 cpg e diferentes níveis de contrastes (indicados à direita de cada traçado). B. Saturação de amplitude da resposta eletrofisiológica em altos contrastes. As amplitudes em altos contrastes (círculos brancos) apresentam valores abaixo daqueles esperados pela função que descreve a amplitude do VEP transiente em baixos contrastes (círculos pretos). C. Dupla tendência de decaimento da amplitude do VEP transiente em função do logaritmo do contraste. A diminuição da amplitude em função do contraste é mais acentuada em altos contrastes (círculos brancos) que em contrastes intermediários e baixos (círculos pretos). D. Comparação da FSC eletrofisiológica (círculos pretos) e FSC psicofísica dinâmica (quadrados brancos), observando-se sua semelhança em freqüências espaciais baixas e intermediárias. As barras verticais indicam o desvio-padrão em torno da média $(\mathrm{n}=6)$.
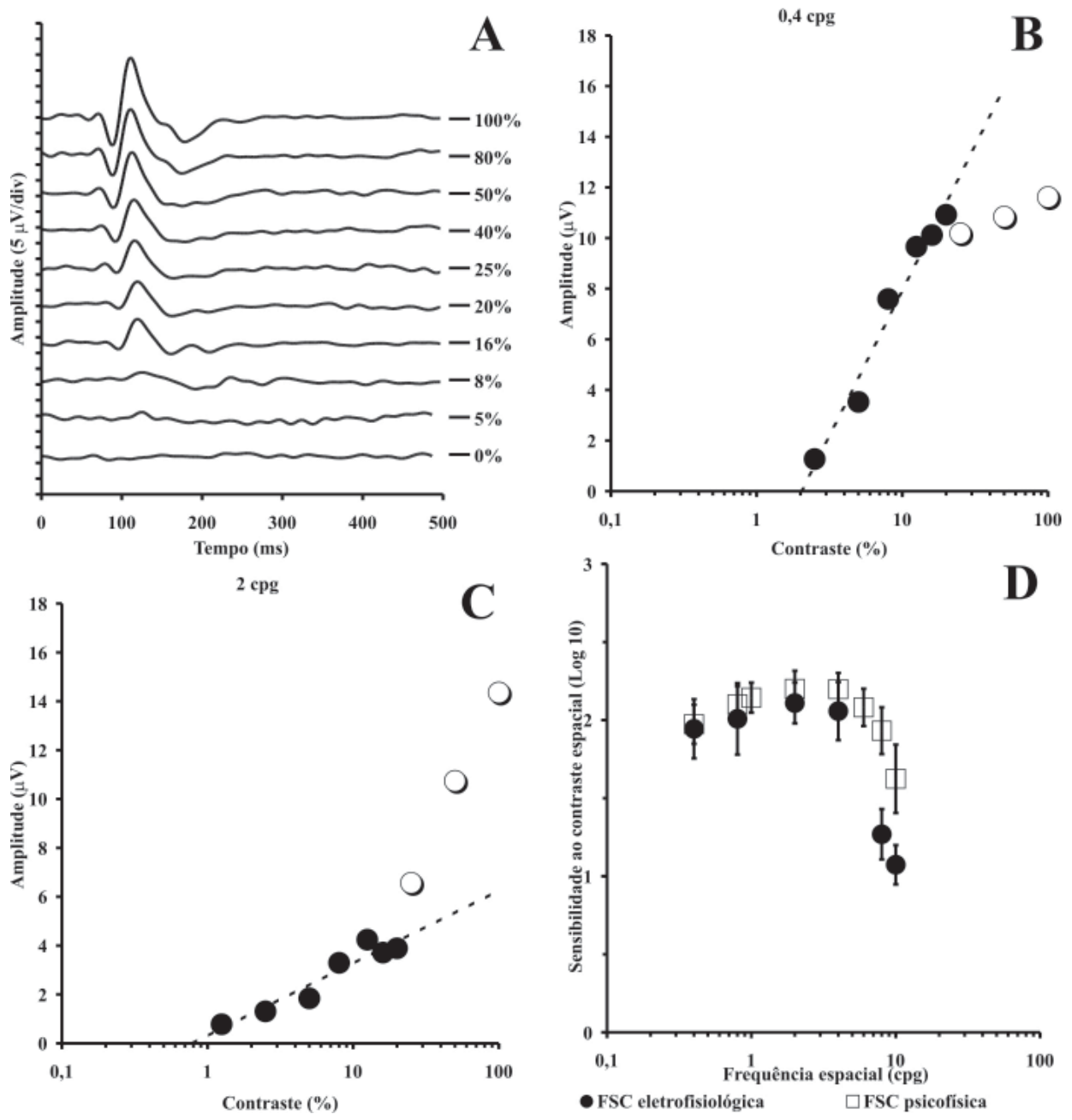


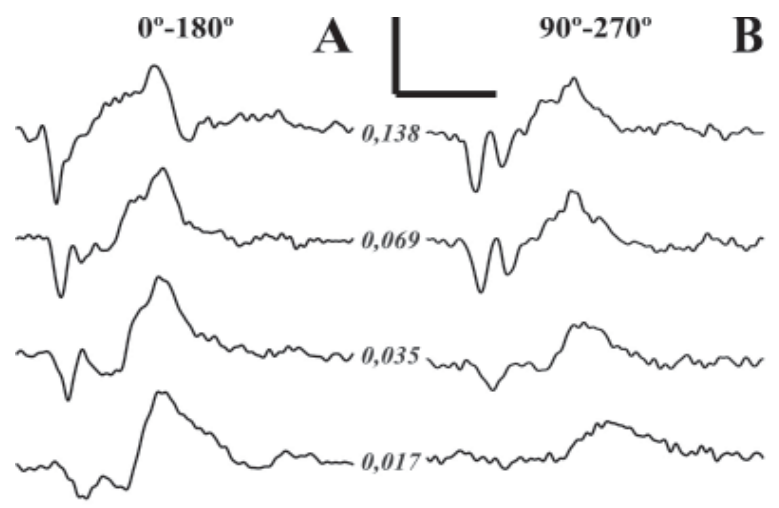

Figura 2. A, B) Registros de VEP transientes obtidos em um sujeito com visão de cores normal para dois eixos ortogonais de cor, $0^{\circ}-180^{\circ}$ e $90^{\circ}-270^{\circ}$. Cada um dos eixos de cor estimula preferencialmente a via de oponência cromática verde-vermelho $\left(0^{\circ}-180^{\circ}\right)(\mathbf{A})$, e azul-amarelo $\left(90^{\circ}-270^{\circ}\right)(\mathbf{B})$. Foram usadas redes senoidais cromáticas isoluminantes em modo de estimulação onset

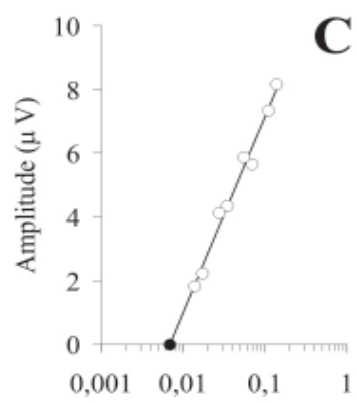

Distância, CIE 1976

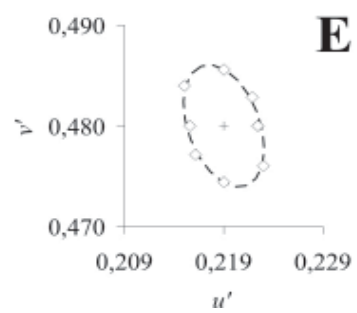

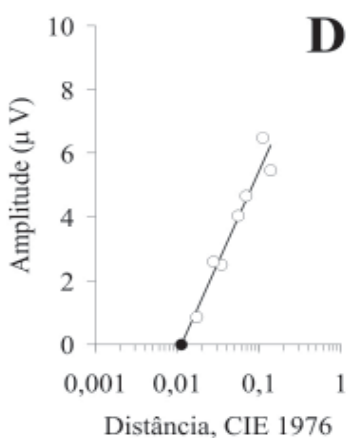

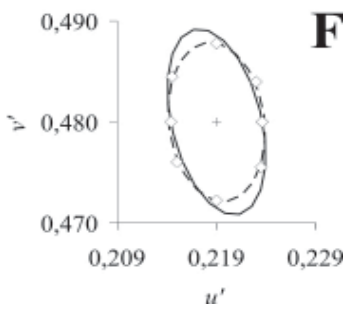
1 offset. Em ambos os eixos, são mostrados quatro registros obtidos em níveis decrescentes de distância cromática (valores centrais em itálico). Existe uma deflexão negativa provocada pelo onset do estímulo, cuja amplitude decresce, acompanhada por um aumento no tempo implícito, à medida que a distância cromática diminui. Escala horizontal e vertical representam, respectivamente, $10 \quad \mu \mathrm{V} \quad \mathrm{e}$ 300 ms. C, D) Amplitude da deflexão negativa do VEP (círculos vazios) em função da distância cromática para os eixos cromáticos mostrados em $\mathbf{A}$ e $\mathbf{B}$, respectivamente. As

retas são funções logarítmicas interpoladas aos dados. A intersecção (círculo cheio) dessa função com o eixo das distâncias cromáticas corresponde ao limiar de discriminação de cores para cada eixo. E) Limiares de discriminação de cores (diamantes vazados) estimados pelo VEP e a elipse de MacAdam correspondente (linha tracejada) para um sujeito com visão de cores tricromática normal. F) Média dos limiares de discriminação de cores (diamantes vazados) estimados pelo VEP e a elipse de MacAdam correspondente (linha tracejada) para um grupo de sujeitos com visão de cores normal $(n=6)$. Para esse mesmo grupo, a elipse de MacAdam psicofísica (linha cheia) obtida com o mesmo estímulo da eletrofisiologia é semelhante em tamanho e forma àquela conseguida com o VEP. 
res (Silveira, Grünert, Kremers, Lee, \& Martin, 2005). As células ganglionares retinianas projetam-se para camadas específicas do núcleo geniculado lateral do tálamo e, daí, as células talâmicas de cada camada também enviam informação para camadas específicas do córtex visual primário. O registro do potencial provocado visual em V1, resultante da estimulação cromática somente é possível porque cerca de $50 \%$ da população de neurônios de V1 responde às variações de cor (Dow \& Gouras, 1973; Gouras, 1974; Yates, 1974).

O processamento de cor no córtex cerebral inicia em circuitos existentes nas camadas 4A e 4C de V1, as quais são ricas na atividade de diversas enzimas do metabolismo oxidativo, inclusive citocromo oxidase, muito utilizada como marcador histoquímico da anatomia cortical (Wong-Riley, 1979a, 1979b). Essas camadas recebem, respectivamente, a projeção de axônios talâmicos K e P, realizam o processamento inicial da informação sobre cor e retransmitem essa informação para outras camadas de V1 e outras áreas corticais. A via M, a qual não contribui para a percepção de cores, tem como porta de entrada no córtex cerebral a camada $4 \mathrm{C} \alpha$ de $\mathrm{V} 1$, outra região rica em atividade enzimática. A partir daí a informação sobre aspectos espaçotemporais acromáticos mediados por essa via é retransmitida para outras camadas de V1 e outras áreas corticais, onde é combinada com os sinais providos por outras vias.

Além do mencionado acima, uma parte importante do processamento cromático ocorre em compartimentos dos estratos mais superficiais de V1, os blobs das camadas 2 e 3, também ricos em atividade enzimática (Wong-Riley, 1979a, 1979b). Os blobs estão alinhados com os centros das colunas de dominância ocular, sendo os compartimentos entre eles, os interblobs, alinhados com as zonas de transição entre uma coluna de dominância ocular e a outra, o que ilustra o arranjo quase cristalino com que forma e função estão representadas no córtex visual primário. Alguns neurônios dos blobs e interblobs possuem propriedades similares aos das vias M, P e K. No entanto, alguma reorganização na informação de cor já pode ser notada. Alguns campos receptivos têm oponência de cores mas não têm uma organização centro-periferia como observado no núcleo geniculado lateral e na retina. Esses neurônios não respondem bem a estímulos luminosos circulares mas, ao invés, respondem selecionando comprimentos de onda independentemente do tamanho do estímulo e exibem oponência de cor (Ts'o \& Gilbert, 1988). Nos blobs existem 
também neurônios com campos receptivos que apresentam um tipo de processamento oponente mais complexo chamado de dupla oponência. As células com dupla oponência de cores possuem campos receptivos organizados em centro e periferia, sendo que a oponência ocorre em ambas as áreas, centro e periferia. Nesse caso, a região central pode estar organizada de modo oponente para vermelho-verde e a periferia para verde-vermelho. Em geral as células com dupla oponência respondem fortemente somente ao contraste cromático e não a contrastes baseados puramente em luminância. Isso ocorre por que as informações de luminância que chegam a esses campos receptivos produzem efeitos que cancelam um ao outro em todo o campo receptivo (Johnson, Hawken, \& Sharpley, 2001; Thorell, De Valois, \& Albrecht, 1984).

A premissa básica na utilização do VEP para estudar a visão de cores humana é que seria possível selecionar as respostas das vias de oponência de cores, manipulando-se o estímulo e analisando componentes específicos da resposta cortical (Engel, Zhang, \& Wandell, 1997; Lennie, Krauskopf, \& Sclar, 1990).

Muitos estudos adotaram estímulos constituídos de redes senoidais cromáticas isoluminantes ou quadradas e com modo de apresentação onset / offset transiente para a obtenção de um VEP de polaridade negativa, o qual é seletivo a redes cromáticas isoluminantes. Com estimulação onset / offset por redes acromáticas o VEP é predominantemente de polaridade positiva e, além disso, outros perfis temporais de estimulação, como a reversão de fase, também originam respostas caracteristicamente positivas, não importando se o estímulo é cromático ou acromático. Essa polaridade da resposta à redes isoluminantes é interpretado como uma ativação seletiva das vias de oponência de cores (Baseler \& Sutter, 1997; Berninger, Arden, Hogg, \& Frumkes, 1989a, 1989b; Gomes, 2005; Gomes et al., 2006; Kulikowski, Murray, \& Parry, 1989; Kulikowski, Robson, \& McKeefry, 1996; Murray, Parry, Carden, \& Kulikowski, 1987; Porciatti, Di Bartolo, Nardi, \& Fiorentini, 1997; Porciatti \& Sartucci, 1999; Rabin, Switkes, Crognale, Schnek, \& Adams, 1994; Suttle, Anderson, \& Harding, 1999). Essa resposta relaciona-se com muitos fenômenos psicofísicos, tal como a degradação do VEP quando se usa estímulos de alta frequiência espacial. Com redes verde-vermelhas, essa degradação ocorre em torno de 8-12 cpg (Kulikowski, Robson, \& Murray, 2002), a qual está bem 
B. D. Gomes, G. S. Souza, A. R. Rodrigues, C. A. Saito, M. Silva-Filho e L. C. L. Silveira

perto do limite de resolução psicofísico para a sensibilidade ao contraste com redes cromáticas isoluminantes (Mullen, 1985).

\section{A utilização do VEP nos estudos de discriminação cromática}

A discriminação cromática pode ser caracterizada e de certo modo quantificada, a partir da especificação dos estímulos através de suas coordenadas de cromaticidade. $\mathrm{O}$ conjunto de cromaticidades (combinações de matizes e saturações de mesma luminância) está representado no diagrama de cromaticidade da CIE (Comission Internationale de L'Eclairage) (Wright, 1998). Esse diagrama pode ser considerado como uma representação do espaço de cor de um indivíduo padrão, tendo sido derivado de medidas psicofísicas. A discriminação de matiz e saturação de duas cromaticidades representam dois casos especiais de discriminação cromática, correspondendo respectivamente à discriminação ao longo das bordas do diagrama de cromaticidade, incluindo o locus espectral e os púrpuras não espectrais, e ao longo de eixos irradiando do branco central em direções às bordas do diagrama. É possível construir um eixo de discriminação puramente cromática escolhendo-se um ponto na região do diagrama correspondente a uma determinada cromaticidade, ligado a um locus correspondendo à outra, desde que ambas estejam com a mesma luminância (Wright, 1941). Para um número de diferentes pontos no plano eqüiluminante do diagrama de cromaticidade, uma série de elipses de discriminação podem ser construídas, as quais representam os limiares de cromaticidade para um número de direções partindo de cada ponto (MacAdam, 1942; Wyszecki \& Stiles, 1982).

Os estudos modernos de discriminação cromática iniciaram com os trabalhos de Yves LeGrand (1949-1994). Usando as elipses de discriminação de cores de MacAdam, LeGrand mostrou que em condições de luminância constante, muito da variação entre as elipses poderia ser explicada considerando duas dimensões dos sinais dos cones: uma na qual os sinais dos cones $\mathrm{S}$ variam isoladamente e outra na qual os sinais dos cones L e M variam de modo inverso mas mantendo uma soma constante entre eles. Essas duas dimensões mais a dimensão em que varia a luminância constituem as direções cardeais do espaço de cores (Krauskopf, Williams, \& Heeley, 1982). 
As elipses de discriminação de cores usadas por LeGrand fornecem uma representação bastante adequada para o estudo da precisão de discriminação de cores. MacAdam idealizou um experimento psicofísico utilizando um colorímetro, onde um observador podia variar a cor da metade de um campo de $2^{\circ}$ de ângulo visual que correspondia a uma determinada distância no diagrama de cromaticidade CIE 1931, com a luminância do campo sendo mantida constante. A outra metade do campo consistia de um estímulo fixo cuja cromaticidade devia ser igualada pelo outro campo através da manipulação do observador segundo a sua sensibilidade. A cromaticidade do estímulo fixo estava localizada na linha de variação onde ocorria a alteração da outra metade do campo. A partir da indicação do indivíduo de que os campos possuíam a mesma cromaticidade, era computada a diferença nas coordenadas entre o estímulo variável e o estímulo fixo. A repetição do experimento em várias direções do espaço de cor permite a determinação das elipses de discriminação de cores ou elipses de MacAdam (MacAdam, 1942; Wyszecki \& Stiles, 1982). Outros experimentos psicofísicos auxiliares foram feitos por MacAdam como os das bordas minimamente distintas com os limiares de discriminação igualmente localizados como pontos de uma elipse de coordenada central igual ao estímulo fixo ao qual se desejava a discriminação limiar (MacAdam, 1942; Siberstein \& MacAdam, 1945; Wyszecki \& Stiles, 1982).

Apesar das elipses de discriminação de cores terem sido criadas a partir de procedimentos psicofísicos, os quais podem fornecer informações preciosas acerca da resposta fisiológica, é extremamente informativo obtê-las de dados que de algum modo independem da resposta voluntária do indivíduo, fornecendo assim uma resposta mais objetiva sobre a discriminação cromática. O VEP pode ser utilizado para esse fim. Entretanto, pouquíssimos trabalhos empregaram tal procedimento.

Macaluso, Baratta, Lamedica e Luani (1994) construíram elipses de discriminação de cores através da estimulação com redes senoidais cromáticas isoluminantes, frequiência temporal de reversão de fase de $8 \mathrm{~Hz}$, registrando VEP de estado estacionário. Macaluso, Baratta, Lamedica e Cordella (1996) estudaram quatro pontos no espaço de cores CIE 1976, correspondendo a quatro limiares de discriminação de cores, usando novamente redes senoidais cromáticas isoluminantes, freqüencia temporal de reversão de fase de $4 \mathrm{~Hz}$ e registrando 
VEP de estado estacionário. Nesses dois trabalhos, os limiares de discriminação cromática foram obtidos através de medidas de amplitude do maior harmônico obtido após a resposta eletrofisiológica ter sido submetida à FFT.

Gomes et al. (2006) estudaram a discriminação de cores medindo os limiares de diferença cromática com VEP transiente em sujeitos com visão tricromática normal (Figura 2) e em um indivíduo deutan. Os estímulos consistiram de redes cromáticas isoluminantes senoidais, $2 \mathrm{cpg}$, construídas de pares de cromaticidades localizadas ao longo de quatro direções diferentes do diagrama de cromaticidade CIE 1976 centrados em dois pontos de referência desse espaço de cores. Eles usaram a fotometria de flicker heterocromático ou HFP (Heterochromatic Flicker Photometry, HFP) para obter a condição de isoluminância para cada sujeito e para cada par de cromaticidades. $\mathrm{O}$ modo de apresentação do estímulo foi onset (300 ms) / offset (700 ms). Gomes et al. (2006) encontraram, como descrito por outros autores, uma deflexão negativa no VEP transiente a qual estava relacionada com a diferença cromática presente no estímulo: à medida que a diferença cromática aumentava, a amplitude do VEP aumentava e a sua latência diminuía. Gomes et al. (2006) relacionaram a amplitude do VEP com a distância no diagrama de cromaticidade CIE 1976 das cromaticidades componentes do estímulo e ajustaram essa função com uma regressão linear a qual, extrapolada para amplitude nula, fornecia o limiar de diferença cromática. O conjunto de limiares assim obtidos foram usados para determinar a elipse de MacAdam em torno de cada um dos dois pontos de referências empregados nesse trabalho. Como esperado, nos tricromatas normais as elipses eram pequenas, pouco alongadas e orientadas na direção tritan (a direção onde um tricromata normal tem um pouco mais de dificuldade em discriminar cores). No sujeito deutan, as elipses eram grandes, extremamente alongadas e orientadas em direção ao locus copunctal deutan. Os limiares obtidos com o VEP são similares àqueles derivados de procedimentos psicofísicos usando estímulos semelhantes e os autores concluíram que a amplitude do VEP transiente em função da diferença cromática (ou contraste de cor) presente num estímulo pode ser usado como medida objetiva do desempenho de sujeitos com visão de cores normal ou alterada (Figura 2). 
Gomes, B. D., Souza, G. S., Rodrigues, A. R., Saito, C. A., Silva-Filho, M., \& Silveira, L. C. L. (2006). Spatial luminance contrast sensitivity and color discrimination measured with transient visual evoked potential. Psicologia USP, 17(4), 63-85.

\begin{abstract}
The Visual Evoked Potential (VEP) is a cortical response obtainable on the scalp. It usually reflects the activity from V1 neurons. It is classified in transient or steady-state, according with the temporal frequency of stimulation. Other stimuli properties evoke a selective activity from different neuronal groups found in V1. This way, VEP have been used to study luminance and chromatic human vision. Several studies used VEP to estimate luminance contrast sensitivity in the spatial frequency domain. More recently, some studies used VEP to measure color discrimination thresholds. The transient VEP shows a good agreement with psychophysical measurements of spatial luminance contrast sensitivity and color discrimination, being a noninvasive method to study vision from subjects with difficulty to perform psychophysical tests.
\end{abstract}

Index terms: Color discrimination. Contraste sensitivity. Visual evoked potentials. Vision estimulation.

Gomes, B. D., Souza, G. S., Rodrigues, A. R., Saito, C. A., Silva-Filho, M., \& Silveira, L. C. L. (2006). La determination de la sensitivité au contraste et de la dicrimination de couleurs au moyen des potentiels évoqués visuels transitoires. Psicologia USP, 17(4), 63-85.

Résumé: Le potenciel évoqué visuel (VEP) est une réponse corticale qui peut être enregistrée à la surface du cuir chevelu. Il reflète l'activité des neurones de V1 et est classifié, selon la fréquence de stimulation temporale, en transitoire ou stationnaire. D'autres propriétés du stimulus semblent provoquer une activité sélective des divers groupes de neurones existants dans V1. Ainsi, le VEP est utilisé pour étudier la vision humaine achromatique et chromatique. Divers travaux ont utilisé le VEP pour estimer la sensibilité au contraste de luminance dans le domaine des fréquences spatiales. Plus récemment, des études ont utilisé le VEP pour mesurer les seuils de discrimination des couleurs. Le VEP transitoire peut compléter les mesures psychophysiques de sensibilité au contraste spatial 
B. D. Gomes, G. S. Souza, A. R. Rodrigues, C. A. Saito, M. Silva-Filho e L. C. L. Silveira

de luminance et de discrimination chromatique et constitue une méthode non envahissante pour étudier la vision des sujets avec des difficultés à réaliser les tests psychophysiques.

Mots-clés: Discrimination des couleurs. Sensibilité de contraste (vision). Potenciels évoqués visuels. Stimulation visuelle.

\section{Referências}

Barlow, H. B. (1972). Single units and sensation: A neuron doctrine for perceptual psychology? Perception, 1, 371-394.

Baseler, H. A., \& Sutter, E. E. (1997). M and P components of the VEP and their visual field distribution. Vision Research, 37, 675-690.

Berninger, T. A., Arden, G. B., Hogg, C. R., \& Frumkes, T. (1989a). Colour vision defect diagnosed by evoked potentials. Investigative Ophthalmology and Vision Science, 30, 290-299.

Berninger, T. A., Arden, G. B., Hogg, C. R., \& Frumkes, T. (1989b). Separable evoked retinal and cortical potentials from each major visual pathway: Preliminary results. Journal of Ophtalmology, 73, 502-511.

Bobak, P., Bodis-Wollner, I., Harnois, C., \& Thornton, J. (1984). VEPs in humans reveal high and low spatial contrast mechanisms. Invesitigative Ophtalmology and Visual Science, $25,980-983$.

Bonds, A. B., Casagrande, V. A., Norton, T. T., \& Debruyn, E. J. (1987). Visual resolution and sensitivity in a nocturnal primate (Galago) measured with visual evoked potentials. Vision Research, 27, 845-857.

Bracewell, R. N. (1986). The fourier transform and its applications (2nd ed.). New York: McGraw-Hill.

Campbell, F. W., \& Kulikowski, J. J. (1972). The visual evoked potential as a function of contrast of a grating pattern. Journal of Physiology (London), 222, 345-356.

Campbell, F. W., \& Maffei, L. (1970). Electrophysiological evidence for the existence of orientation and size detectors in the human visual system. Journal of Physiology (London), 207, 635-652.

Campbell, F. W., \& Robson, J. G. (1968). Application of Fourier analysis to the visibility of gratings. Journal of Physiology (London), 197, 551-566.

Cannon, M. W., Jr. (1983a). Contrast sensitivity: Psychophysical and evoked potential methods compared. Vision Research, 23, 87-95. 
Estimativa da Sensibilidade ao Contraste Espacial de Luminância e Discriminação...

Cannon, M. W., Jr. (1983b). Evoked potential contrast sensitivity in the parafovea: Spatial organization. Vision Research, 23, 1441-1449.

Chen, S., Wu, L., \& Wu, D. (1990). Objective measurement of contrast sensitivity using the steady-state visual evoked potential. Documenta Ophthalmologica, 75, 145-153.

De Valois, R. L., Yund, E. W., \& Hepler N. (1982). The orientation and direction selectivity of cells in macaque visual cortex. Vision Research, 22, 531-544.

Dow, B. M., \& Gouras, P. (1973). Color and spatial specificity of single units in Rhesus monkey foveal striate cortex. Journal of Neurophysiology, 36, 79-100.

Engel, S., Zhang, X., \& Wandell, B. (1997). Color tuning in human visual cortex measured with functional magnetic resonance imaging. Nature, 388, 68-71.

Gomes, B. D. (2005). Determinação das elipses de discriminação de cores através da estimulação com redes senoidais cromáticas e registro do potencial cortical provocado visual transiente: comparação com resultados obtidos com métodos psicofísicos. Dissertação de Mestrado, Programa de Pós-graduação em Neurociências e Biologia Celular, Universidade Federal do Pará, Belém, PA.

Gomes, B. D., Souza, G. S., Rodrigues, A. R., Saito, C. A., Silveira, L. C. L., \& da Silva Filho, M. (2006). Normal and dichromatic color discrimination measured with transient VEP. Visual Neuroscience, 23, 617-627.

Gouras, P. (1974). Opponent colour cells in different layers of foveal striate cortex. Journal of Physiology (London), 199, 533-547.

Harris, L., Atkinson, J., \& Braddick, O. (1976). Visual contrast sensitivity of a 6 month old infant measured by the evoked potential. Nature, 264, 570-571.

Hendry, S. H. C., \& Reid, R. C. (2000). The koniocellular pathway in primate vision. Annual Review of Neuroscience, 23, 127-153.

Hjorth, B. (1975). An-online transformation of EEG scalp potentials into orthogonal source derivation. Electroencephalography and Clinical Neurophysiology, 39, 526-530.

Johnson, E. N., Hawken, M. J., \& Shapley, R. (2001). The spatial transformation of color in the primary visual cortex of the macaque monkey. Nature Neuroscience, 4, 409-416.

Kaplan, E., \& Shapley, R. M. (1986). The primate retina contains two types of ganglion cells, with high and low contrast sensitivity. Proceedings of the National Academy of Science of the U.S.A., 83, 2755-2757.

Katsumi, O., Tanino, T., \& Hirose, T. (1985). Measurement of contrast sensitivity function using pattern-reversal visual evoked responses. Graefes Archives of Clinical and Experimental Opthalmology, 223, 190-195.

Krauskopf, J., Williams, D. R., \& Heeley, D. W. (1982). Cardinal directions od color space. Vision Research, 22, 1123-1131. 
B. D. Gomes, G. S. Souza, A. R. Rodrigues, C. A. Saito, M. Silva-Filho e L. C. L. Silveira

Kulikowski, J. J., Murray, I. J., \& Parry, N. R. A. (1989). Electrophysiological correlates of chromatic opponent and achromatic stimulations in man. In B. Drum \& G. Verriest (Eds.), Colour vision deficiences, 9: Documenta ophthalmologica proceedings (pp. 145153). Dordrecht: Kluwer Academic.

Kulikowski, J. J. , Robson, A. G., \& Murray, I. J. (2002). Scalp VEPs and intra-cortical responses to chromatic and achromatic stimuli in primates. Documenta Ophthalmologica, 105, 243-279.

Kulikowski, J. J., Robson, A. G., \& Mckeefry, D. J. (1996). Specificity and selectivity of chromatic visual evoked potentials. Vision Research, 36, 3397-3401.

Lennie, P., Krauskopf, J., \& Sclar, G. (1990). Chromatic mechanisms in striate cortex of macaque. Journal of Neuroscience, 10, 649-669.

Lopes de Faria, J. M., Katsumi, O., Arai, M., \& Hirose, T. (1998). Objective measurement of contrast sensitivity function using contrast sweep visual evoked responses. British Journal of Ophtalmology, 82, 168-173.

Macaluso, C., Baratta, G., Lamedica, A., \& Cordella, M. (1996). Color discrimination along the cardinal chromatic axes with VECPs as an index of function of the parvocellular pathway. Correspondence of intersubject and axis variations to psychophysics. Electroencephalography and Clinical Neurophysiology, 100, 12-17.

Macaluso, C., Baratta, G., Lamedica, A., \& Luani, D. (1994). Electrophysiological determination of MacAdam ellipses. A VEP study. Investigative Ophthalmology and Visual Science, 35, 1977.

MacAdam, D. L. (1942). Visual sensitivities to color differences in daylight. Journal of the Optical Society of America, 32, 247-274.

Mullen, K. T. (1985). The contrast sensitivity of human color vision to red-green and blueyellow chromatic gratings. Journal of Physiology (London), 359, 381-409.

Murray, I. J., Parry, N. R., Carden, D., \& Kulikowski, J. J. (1987). Human visual evoked potentials to chromatic and achromatic gratings. Clinical and Vision Science, 3, 231-244.

Nakayama, K., \& Mackeben, M. (1982). Steady state visual evoked potentials in the alert primate. Vision Research, 22, 1261-1271.

Norcia, A. M., Tyler, C. W., Hamer, R. D., \& Wesemann, W. (1989). Measurement of spatial contrast sensitivity with the swept contrast VEP. Vision Research, 29, 627-637.

Odom, J. V., Bach, M., Barber, C., Brigell, M., Marmor, M. F., Tormene, A. P. et al. (2004). Visual evoked potentials standard. Documenta Ophtalmologica, 108, 115-123.

Oliveira, A. G. F., Costa, M. F., de Souza, J. M., \& Ventura, D. F. (2004). Contrast sensitivity threshold measured by sweep-visual evoked potential in term and preterm infants at 3 and 10 months of age. Brazilian Journal of Medical and Biological Research, 37, 1389-1396. 
Estimativa da Sensibilidade ao Contraste Espacial de Luminância e Discriminação...

Orban, G. A., \& Orban, L. C. (1985). Rapid contrast sweeping of steady-state visual evoked potentials: A new method for investigation of the visual system. Documenta Ophthalmologica, 61, 7-16.

Porciatti, V., Di Bartolo, E., Nardi, N., \& Fiorentini, A. (1997). Responses to chromatic and luminance contrast in glaucoma: A psychophysical and electrophysiological study. Vision Research, 37, 1975-1987.

Porciatti, V., \& Sartucci, F. (1999). Normative data for onset VEPs to red-green and blueyellow chromatic contrast. Clinical Neurophysiology, 110, 772-781.

Rabin, J., Switkes, E., Crognale, M., Schnek, M. E., \& Adams, A. J. (1994). Visual evoked potentials in three-dimensional color space: Correlates of spatio-chromatic processing. Vision Research, 34, 2657-2671.

Regan, D. (1966). An effect of stimulus colour on average steady-state potentials evoked in man. Nature, 210, 1056-1057.

Regan, D. (1982). Comparison of transient and steady-states methods. Annals of the New York Academy of Science, 388, 45-71.

Regan, D., \& Spekreijse, H. (1986). Evoked potentials in vision research: 1961-1985. Vision Research, 26, 1461-1480.

Rudvin, I., Valberg, A., \& Kilavik, B. E. (2000). Visual evoked potentials and magnocellular and parvocellular segregation. Visual Neuroscience, 17, 579-590.

Seiple, W. H., Kupersmith, M. J., Nelson, J. I., \& Carr, R. E. (1984). The assessment of evoked potential contrast thresholds using real-time retrieval. Investigative Ophthalmology and Visual Science, 25, 627-631.

Schade, O. H. (1956). Optical and photoelectric analog of the eye. Journal of the optical Society of America, 46, 721-739.

Siberstein, L., \& MacAdam, D. L. (1945). The distribution of colour matchings around a color center. Journal of Optical Society of America, 35, 32-39.

Silveira, L. C. L., Picanço-Diniz, C. W., Sampaio, L. F. S., \& Oswaldo-Cruz, E. (1989). Retinal ganglion cell distribution in the Cebus monkey: A comparison with the cortical magnification factors. Vision Research, 29, 1471-1483.

Silveira, L. C. L., Grünert, U., Kremers, J., Lee, B. B., \& Martin, P. R. (2005). Comparative anatomy and physiology of the primate retina. In J. Kremers (Ed.), The primate visual system: A comparative approach (pp. 127-160). Chichester, England: John Wiley \& Sons.

Sincich, L. C., \& Horton, J. C. (2005). The circuitry of V1 and V2: Integration of color, form, and motion. Annual Review of Neuroscience, 28, 303-326.

Souza, G. S. (2005). Avaliação da sensibilidade ao contraste de luminância humana através do potencial cortical provocado visual transiente - Comparação com resultados 
obtidos psicofisicamente. Dissertação de Mestrado, Programa de Pós-graduação em Neurociências e Biologia Celular, Universidade Federal do Pará, Belém, PA.

Souza, G. S., Gomes, B. D., Saito, C. A., Silveira, L. C. L., \& da Silva Filho, M. (2006). Human achromatic spatial contrast sensitivity measured with transient visual evoked cortical potential: Comparison with psychophysics measurements. Investigative Ophthalmology and Visual Science, 47, E-Abstract No. 5379/B9999.

Strasburger, H., Rentschler, I., \& Scheidler, W. (1986). Steady-state pattern vep uncorrelated with suprathreshold contrast perception. Human Neurobiology, 5, 209-211.

Strasburger, H., Remky, A., Murray, I., Hadjizenonos, C., \& Rentschler, I. (1996). Objetive measurement of contrast sensitivity and visual acuity with the steady-state visual evoked potential. German Journal of Ophtalmology, 5, 42-52.

Suttle, C. M., Anderson, S. J., \& Harding, G. F. A. (1999). A longitudinal study of visual evoked responses to tritan stimuli in humans infants. Optometry and Vision Science, 74, 717-725.

Thorell, L. G., De Valois, R. L., \& Albrecht, D. G. (1984). Spatial mapping of monkey V1 cells with pure color and luminance stimuli. Vision Research, 24, 751-769.

Tobimatsu, S., Kurita-Tashima, S., Nakayama-Hiromatsu, M., \& Kato, M. (1993). Effect of spatial frequency on transient and steady-state VEPs stimulation with checkerboard, square-wave grating and sinusoidal grating patterns. Journal of Neurological Science, $118,17-24$.

Ts'o, D. Y., \& Gilbert, C. D. (1988). The organization of chromatic and spatial interactions in the primate striate cortex. Journal of Neuroscience, 8, 1712-1727.

Westheimer, G. (1960). Modulation thresholds for sinusoidal light distributions on the retina. Journal of Physiology (London), 152, 67-74.

Wyszecki, G., \& Stiles, W. S. (1982). Color science: Concepts and methods, quantitative data and formulae. New York: John Wiley \& Sons.

Wong-Rilley, M. T. T. (1979a). Columnar cortico-cortical interconections within the visual system of the squirrel and macaque monkey. Brain Research, 162, 201-217.

Wong-Rilley, M. T. T. (1979b). Changes in the visual system of monocularly sutured or enucleated cats demonstrable with cytochrome oxidase histochemistry. Brain Research, $171,11-28$.

Wright, W. D. (1941). The sensitivity of the eye to small colour differences. Proceedings of Physical Society (London), 53, 96-112.

Wright, W. D. (1998). The origins of the 1931 CIE System. In P. K. Kayser \& R. M. Boynton (Eds.), Human color vision (pp. 534-543). Washington, DC: Optical Society of America. 
Estimativa da Sensibilidade ao Contraste Espacial de Luminância e Discriminação...

Whittaker, S. G., \& Siegfried, J. B. (1983). Origin of wavelets in the visual evoked potential. Electroencephalography and Clinical Neurophysiology, 55, 91-101.

Yates, J. T. (1974). Chromatic information processing in the foveal projection (area striata) of unanesthetized primate. Vision Research, 14, 163-173.

Recebido em: 18/12/2006

Aceito em: 21/12/2006 